\title{
Laparoscopia quirúrgica en ginecología: entrenamiento, certificación y acreditación
}

\author{
Jaime Saavedra Saavedra*
}

\section{RESUMEN}

En la actualidad es obvio e inevitable idear e implementar un protocolo racional bien estructurado que nos permita obtener un entrenamiento, certificación y acreditación en cirugía endoscópica.

Hoy día el buen nombre del cirujano y de la institución quirúrgica depende del entrenamiento y acreditación en endoscopia quirúrgica ginecológica y del deseo por alcanzar la excelencia.

Si nosotros como cirujanos ginecólogos endoscopistas no establecemos rápidamente la pautas, otras partes interesadas llámese gobierno, medicina prepagada, empresas prestadoras de salud, se encargaran de establecer los procedimientos unilateralmente.

PALABRAS CLAVES: Laparoscopia, enseñanza, certificación, acreditación.

\section{SUMMARY}

The need for devising and implementing a rational and structured training, certification, and credentialing protocol in operative endoscopy has become obvious and anavoidable.

Training and credentialing in gynecologic operative endoscopy today depends on the surgeon's and the surgical institutio's goodwill and desire for excelIence.

If we as the concerned practitioners do not rapidly establish such guidelines, other interested parties, namely governments and third party payers will and are proceeding to do so unilateralIy.

KEY WORDS: Laparoscopy, training, certification, crendentialing.

La realización de procedimientos endoscópicos en ginecología no es reciente. Desde 1940 se realiza en forma regular. En la década de los años de 1970 un número considerable de ginecoobstetras se inicia en este campo a través de las campañas masivas de planificación familiar en las que se realizaba ligadura de trompas por este método. La práctica de la endoscopia en ese momento la realizaba un reducido número de especialistas que recibían adiestramiento supervisado en cirugía laparoscópica en centros especializados, que además de hacer ligaduras de trompas hacían laparoscopias diagnósticas.

La práctica de laparoscopia quirúrgica estaba restringida a unos cuantos procedimientos de complejidad baja

\footnotetext{
Profesor Titular. Departamento de Ginecología y Obstetricia Universidad del Valle-Cali, Colombia. Jefe del servicio de Infertilidad y Endocrinología, Hospital Universitario del Valle. Director del Programa del Entrenamiento en Cirugía Endoscópica Ginecológica. Clínica Los Andes-Cali-Colombia. Directos General del Centro de Biomedicina Reproductiva -FECUNDAR-. CaliColombia. Presidente Sociedad Colombiana de Endoscopia.
}

y solo en unos cuantos centros en el mundo se practicaba la cirugía avanzada a partir de 1988.

Después de la descripción de las técnicas de histerectomía y la colecistectomía laparoscópica, la cirugía endoscópica sufrió un avance explosivo y su práctica se extendió rápidamente a todas las áreas de cirugía general y la ginecoobstetricia.

Las razones de esta gran expansión incluyen: las ventajas que la laparoscopia ofrece al paciente, como es el menor dolor, menos adherencias y recuperación rápida, versatilidad en la técnica, la presión que ejercen las compañías fabricantes de equipos endoscópicos sobre los cirujanos, e incluso la creciente petición de las pacientes para que la cirugía que se les practique sea a través de la laparoscopia.

Por otra parte, si bien la cirugía endoscópica tiene grandes ventajas, debe ser practicada por cirujanos capacitados, y su realización no esta exenta de riesgos. A pesar que su índice de complicaciones es bajo, su práctica puede llevar a serios problemas incluso mortales (1-4).

En el reciente caos por practicar cirugía endoscópica comenzó a aparecer en forma frecuente iatrogenia y en muchos de los casos esta fue grave. 
En ocasiones, el cirujano emprendía la realización de una laparoscopia operatoria después de tomar un curso teórico práctico de apenas dos días de duración, sin práctica previa en pacientes.

Un entrenamiento adecuado, con la certificación de capacidad, y su acreditación correspondiente es importante para asegurar una alta calidad del servicio prestado, maximizando un resultado exitoso, con una mínima morbilidad. Además, la documentación de unas habilidades apropiadas, es esencial para la protección médico legal del hospital o clínica prestadora del servicio, del personal auxiliar del quirófano y del cirujano. Si bien las complicaciones son un riesgo aceptado en endoscopia o en cualquier cirugía, la corroboración del entrenamiento y habilidades del cirujano en estas técnicas podrían minimizar la responsabilidad legal en el caso de enfrentar esta eventualidad.

Es una falacia común entre los médicos, el creer que la implementación de un proceso uniforme y claro de acreditación para la endoscopia quirúrgica en el hospital o clínica, resulta en un riesgo aumentado de problemas legales.

La realidad es que los hospitales, clínicas y su personal usualmente es encontrado responsables por los jueces cuando se presenta un caso negativo, por no haber establecido las medidas adecuadas para tener un control de calidad razonable.

El entrenamiento y acreditación del cirujano ginecológico endoscopista se ha convertido en una necesidad urgente e inmediata en nuestra especialidad (5-6).

La urgencia se debe a que instituciones no médicas están dando rápidamente los pasos para implementar las pautas de acreditación. Ejemplo de ello es que algunas empresas prestadoras de servicios médicos ayudadas por médicos sin mayor experiencia en la materia han promulgado pautas sobre los niveles de complejidad de los procedimientos endoscópicos, sin evaluar el nivel de entrenamiento del cirujano que los va a realizar, y sin tener en cuenta si el hospital o clínica donde se realiza cirugía endoscópica a determinado a quienes se le da la prerrogativa de realizar este tipo de procedimientos, y si la institución reúne las condiciones en locación e instrumental para ejecutar esta actividad. Desafortunadamente un entrenamiento pobre e inadecuado y la no-certificación tiene un efecto negativo y potencialmente fatal, como se ha visto últimamente por un incremento en muertes y complicaciones serias en pacientes en las cuales se les ha realizado cirugía endoscópica.

A continuación describimos el plan general que antecede al entrenamiento y certificación en cirugía endoscópica ginecológica, y sugerimos el perfil de entrenamiento que nos va asegurar una pericia y un expertismo óptimo entre los cirujanos ginecológicos endoscopistas (7).

\section{Esquemas de entrenamiento, certificación, y acreditación}

El entrenamiento se refiere al proceso por el cual el conocimiento y la habilidad son adquiridos; la certificación se refiere al proceso de la documentación de la habilidad o expertismo adquirido; y acreditación hace referencia al proceso por el cual el cuerpo directivo del hospital o clínica reconoce el entrenamiento y capacidad del cirujano y le confiere a él o ella el privilegio para realizar estos procedimientos dentro de los confines del hospital o clínica.

Debido a que nuestro país no había un ente regulador de los procesos antes anotados, en 1995 se unieron la Sociedad Colombiana de Médicos Endoscopistas y la Sociedad Colombiana de Ginecología y Obstetricia para crear el Consejo de Acreditación para la Endoscopia Ginecológica; la misión de este es elevar los estándares de calidad de la cirugía endoscópica realizada por los ginecólogos.

Los directores de este Consejo de Acreditación son los endoscopistas más notables y con mayor reconocimiento en el país.

Este consejo de acreditación regulará la práctica de la endoscopia ginecología con base en la autoridad moral de sus directores y en la fuerza que le otorgan la Sociedad Colombiana de Médicos Endoscopistas y la Sociedad Colombiana de Ginecología y Obstetricia

Para ser reconocido por este consejo el aspirante debe ser ginecoobstetra certificado y probar haber realizado 50 casos de laparoscopia operatoria avanzada y 20 casos de histeroscopia operatoria.

Esta norma es obligatoria en todo el territorio colombiano.

Se ha establecido que la laparoscopia operatoria debe realizarse en unidades quirúrgicas de segundo y tercer nivel. El equipo mínimo necesario para la realización de estos procedimientos es:

1. Equipo de cámara y video

2. Laparoscopio de $10 \mathrm{~mm}$ de $0^{\circ}$ o de $12 \mathrm{~mm}$ con canal operatorio y de $10^{\circ}$

3. Insuflador automático de $\mathrm{CO} 2$

4. Equipo de aspiración e irrigación

5. Equipo de anestesia con oximetro de pulso y cápnografo

6. Unidad electroquirúrgica, o de radiofrecuencia o láser quirúrgico

7. Instrumental adecuado para realizar la cirugía endoscópica

8. Disponibilidad de equipo para laparotomía en caso que se necesite.

Además, los sitios donde se practique la cirugía laparoscópica deben tener un comité de laparoscopia integrado por un jefe de cirugía, cirujanos laparoscopistas certificados por el consejo de la especialidad, anestesiólogo, y representante administrativo. Todos los cirujanos que practiquen cirugía laparoscópica, deben estar certificados por el consejo de la especialidad respectiva y haber realizado un curso de entrenamiento formal teórico práctico de por los menos 2 meses de duración impartidos por instituciones reconocidas.

El entrenamiento, certificación y acreditación se pueden basar en una variedad de esquemas, diferenciándose estos en el grado de especificidad. Tabla 1

No obstante, estos tres pasos forman parte del mismo proceso y se debería idealmente seguir el mismo esquema.

La acreditación se puede asignar sobre la base del expertismo en endoscopia quirúrgica como un conjunto. 
Tabla 1

VARIOS ESQUEMAS DE ENTRENAMIENTO, CERTIFICACIÓN Y ACREDITACIÓN

Para endoscopia quirúrgica en conjunto

Para endoscopia quirúrgica e y histeroscopia por separado

Por nivel de habilidad, para laparoscopia quirúrgica y

histeroscopia por separado

Por tipo de procedimiento

El cirujano es experto o no experto en toda la técnica de laparoscopia quirúrgica.

Para el Colegio Americano de Obstetricia y Ginecología (8) y la Asociaci6n Americana de Ginec6logos Endoscopistas (9) dicho esquema es de aceptación general.

En el segundo, el entrenamiento y la asignación de privilegios para realizar procedimientos puede ser separado en aquellos para laparoscopia operatoria y en aquellos para histeroscopia operatoria.

Este tipo de esquema fue previamente recomendado por el Colegio Americano de Obstetricia y Ginecología (10-11).

Un tercer esquema es el de un entrenamiento más intensivo con certificaci6n del mismo basado en procedimientos específicos, documentando separadamente las habilidades para realizar histerectomía asistida por laparoscopia, neurectomía presacra, neosalpingostomía, etc.

Nosotros, en la Sociedad Colombiana de Médicos Endoscopistas preferimos un esquema alterno, de complejidad intermedia, basado en agrupar los procedimientos laparoscópicos o histeroscópicos de acuerdo al nivel de habilidad requerida para realizarlos, siendo esto aceptado por la mayoría de las agrupaciones líderes en el campo (7). Tabla 2 y 3.

Creemos que lógica de este esquema es óptimo porque él sigue de forma natural la obtención de habilidades quirúrgicas, y no requiere de rectificaciones continuas, en la medida que se describen nuevas aplicaciones para la endoscopia quirúrgica ginecológica, y el que hace el entrenamiento puede realmente obtener suficiente experiencia en un tiempo razonable para documentar su competencia.

El sistema de acreditación por nivel de habilidades ha sido adoptado por instituciones como la Sociedad Canadiense de Obstetras y Ginecólogos (SOGC) y la Sociedad de Cirujanos de Cirugía Reproductiva (SRS) de la Sociedad Americana de Medicina Reproductiva (12).

\section{Entrenamiento en Endoscopia Quirúrgica}

Entrenamiento se refiere a la parte de proceso por el cual el estudiante adquiere el suficiente conocimiento y habilidades para:

1. Seleccionar en forma apropiada los pacientes para el tratamiento quirúrgico, excluyendo a aquellos quienes se podrían beneficiar de una intervención no quirúrgica.
Tabla 2

NIVELES DE HABILIDADES SUUGERIDAS PARA ENTRENAMIENTO, CERTIFICACIÓN, Y ACREDITACIÓN EN LAPAROSCOPIA QUIRÚRGICA

Nivel 1 (laparoscopia quirúrgica Básica)

Ablación o remoción de endometriosis leve a moderada, incluyendo endometriomas

$<3$ de cm en diámetro

Salpingo-ovariólisis de adherencias leves

Salpinguectomía subtotal (coagulación y corte)

Fulguración superficial del ovario (endometriosis mínima, adherencias velamentosas)

Punción de quistes benignos de ovario

Sección de ligamentos uterosacros

Nivel 2 (Laparoscopia quirúrgica avanzada)

Adhesiolisis

Adherencias intestinales densas

Adherencias intestinales medias y superiores

Miomectomía

Ooforectomía y remoción de endometrioma

Salpingo- ooforectomía

Salpinguectomía

Tratamiento del embarazo ectópico

Neosalpingostomía y fimbrioplastia

Lisis de adherencias moderadas y severas peri tubáricas y ováricas

Apendicectomía

Nivel 3 (Laparoscopia quirúrgica para expertos)

Histerectomía total (las arterias uterinas son incluidas laparoscópicamente)

Histerectomía subtotal

Disección retroperitoneal

Suspensión de vejiga y uretra

Neurectomía presacra

Varicocelectomía pélvica

Reconstrucción del piso pélvico

Linfadennectomía

Tabla 3

NIVELES DE HABILIDADES SUGERIDAS PARA ENTRENAMIENTO, CERTIFICACIÓN Y ACREDITACIÓN EN HISTEROSCOPIA OPERATORIA

Nivel 1 (Histeroscopia quirúrgica básica)

Remoción de pólipos, pequeños miomas y extracción de DIU Biopsia de endometrio dirigida

Lisis de sinequias mínimas a moderadas

Nivel 2 (histeroscopia quirúrgica avanzada)

Lisis de sinequias severas con obliteración de la cavidad uterina

Ablación endometrial

Resección de miomas grandes 
2. Seleccionar el tipo de procedimiento, incluyendo la forma de realizarlo (ejemplo, endoscópicamente o por laparotomía)

3. Aconsejar y obtener el consentimiento del paciente

4. Seleccionar los instrumentos necesarios

5. Realizar el procedimiento

6. Manejar y evitar las complicaciones intraoperatorias

7. Manejo del postoperatorio en curso

8. Documentación de los hallazgos, presentación de estos al paciente, indicaciones y consejos al respecto.

El aspirante a endoscopista quirúrgico debe demostrar conocimiento y habilidades apropiadas para llevar a cabo laparoscopia diagnóstica e histeroscopia. Para el ginecólogo recién egresado estas técnicas deben ser incorporadas durante el entrenamiento de su residencia. Para los otros estas habilidades se deben adquirir en medio de una práctica activa e independiente.

El entrenamiento en endoscopia quirúrgica se puede dividir en tres partes, una fase específicamente didáctica, una observacional, y una tutorial.

\section{Fase didáctica}

En esta fase es importante tener claro los objetivos del aprendizaje, y se dividen en dos grupos: en el primero de ellos el estudiante debe alcanzar un expertismo y en el segundo el estar bien informado.

Ser experto es ser competente de realizar una tarea dada o demostrar una técnica asignada, estar bien informado es ser capaz de demostrar comprensión. La siguiente es una lista de objetivos didácticos que sirven de marco de referencia para llevar acabo esta fase.

\section{Evaluación preoperatoria}

A. Objetivos prácticos

1. Demostrar la habilidad para obtener un consentimiento informado que incluye indicación y diagnóstico, potenciales beneficios, potenciales riesgo, alternativas médicas o quirúrgicas.

2. Demostrar la habilidad para aconsejar a los pacientes, mirando los riesgos y beneficios de la cirugía endoscópica.

B. Objetivos didácticos

1. Estar familiarizado con la patología clínica y el examen clínico para llegar un diagnóstico pertinente para determinar el procedimiento a seguir.

2. Estar familiarizado con la necesidad de realizar una preparación prequirúrgica (preparación de intestino, antibióticos etc.)

3. Estar familiarizado con alternativas médicas o quirúrgicas pertinentes a un procedimiento específico.

4. Conocer las contraindicaciones, relativas y absolutas, para realizar un procedimiento endoscópico quirúrgico.

Este material a menudo se piensa que es mundano y fácil de obtener durante la cirugía. Se prefiere que cada estudiante tenga un conocimiento profundo de este material antes de realizar cirugía. La demostración de la adquisición de estos conocimientos da al estudiante confianza y seguridad.

\section{Instrumentación y equipos}

A. Objetivos prácticos

1. Demostrar la habilidad para ensamblar y desensamblar los instrumentos usados procedimientos endoscópicos básicos.

2. Demostrar la correcta conexión del sistema cámara y video al laparoscopio, incluyendo la integración de monitor, video, impresora y grabadora

3. Demostrar la habilidad para detectar y solucionar los problemas más comunes que se presentan con el sistema de video durante los procedimientos quirúrgicos.

B. Objetivos didácticos

1. Conocer la función y aplicación de los instrumentos utilizados con más frecuencia en la institución de entrenamiento.

2. Conocer la física básica de la óptica del laparoscopio

3. Conocer las presiones correctas a las cuales debe funcionar tanto el insuflador como la máquina de succión aspiración.

\section{Anatomía básica y técnicas de insuflación}

A. Objetivos prácticos

1. Demostrar la adecuada posición prequirúrgica del paciente en la laparoscopia rutinaria

2. Demostrar la técnica para realizar el neumoperitoneo mediante la inserción de la aguja de Verres o trocar directo.

3. Demostrar la técnica adecuada de inserción de trocares auxiliares

4. Demostrar la capacidad de identificar laparoscópicamente la anatomía normal y anormal de la pared abdominal, de los órganos abdominales y pélvicos.

B. Objetivos didácticos

1. Conocer otras alternativas aceptables de inserción de la aguja de insuflación para establecer el neumoperitoneo.

2. Conocer la técnica correcta para la laparoscopia abierta y la inserción del trocar directo, incluyendo la indicación y contraindicaciones de esta técnica.

Muchos de estos objetivos son demostrados durante la revisión de videos con el estudiante como observador. Aunque estos objetivos parecen estar orientados hacia la técnica, ellos se deben complementar con lecturas apropiadas.

\section{Complicaciones}

La información requerida para minimizar y manejar las complicaciones relacionadas con la endoscopia quirúrgica debe ser entendida por el médico en forma clara. Es difícil construir modelos que demuestren con precisión las complicaciones. Por tanto estos objetivos son primariamente didácticos. El estudiante debe ser capaz de describir como reconocer, minimizar la incidencia de complicaciones secundarias a la aguja de insuflación y la inserción de los trocares. 
A. Lesiones vasculares

1. Vasos de la pared abdominal

2. Vasos intraabdominales (ej: omento, mesentéricos, peritoneales)

3. Vasos retroperitoneales (ej: aorta, vena cava, arteria y venas ilíacas)

B. Lesiones entéricas

1. Estómago, intestino delgado y grueso

2. Vejiga y uréter.

3. Utero, ovario y trompas de Falopio.

Estos puntos se refieren a todos los tipos de lesiones: láser, térmico, eléctrico, aplastamiento, laceración y perforación.

C. Cardiopulmonar

1. Arritmias, hipercapnia, complicaciones anestésicas

2. Sobre carga de líquidos

3. Edema pulmonar

4. Embolismo

D. Postquirúrgico

1. Hernia incional

2. Hemorragia tardía

\section{Técnicas hemostáticas e incisionales.}

La aplicación de diferentes fuentes, de energía así como las diferentes técnicas de sutura y ligadura son de gran importancia en la laparoscopia quirúrgica.

Debido a que muchas de las instituciones de entrenamiento no tienen todas las facilidades, el estudiante debe ser conocedor de todas las técnicas, pero debe obtener las habilidades en las técnicas disponibles en la institución donde se encuentre.

La elección de una técnica incisional en particular es a menudo operador dependiente, por esta razón, el estudiante debería ser experto en más de una técnica. Esta regla se aplica a las técnicas hemostáticas. El énfasis debe ser, alcanzar versatilidad y destreza. Para cada técnica o aplicación de energía, el estudiante debe ser capaz de describir los instrumentos necesarios, la técnica a aplicar, efecto sobre el tejido, limitaciones, minimización de complicaciones y su manejo, costo efectividad cuando se compara dos técnicas comparables.

A. Disección cortante

1. Instrumentación

2. Técnica y aplicación

B. Técnicas de sutura y ligadura

1. Nudos extracorpóreos.

2. Sutura intracorporea

3. Sistema de ligadura pre cargada

4. Clips y cosedoras hemostáticas

C. Electrocirugía

1. Física básica de la electricidad

2. Efecto tisular: fulguración, coagulación, desecación, corte

3. Circuitos eléctricos: bipolar, unipolar,

4. Tipos de Onda: modulada, no modulada, mixta

5. Complicaciones: acople capacitivo, acople directo, fallas del electrodo a tierra

D. Láser

1. Física: poder de densidad, producción del láser, longitud de onda.
2. Efecto sobre el tejido

3. Comparación de los diferentes tipos de láseres

4. Seguridad

Como parte de las sesiones didácticas, el estudiante debe desarrollar en el laboratorio las habilidades necesarias para ejecutar una endoscopia quirúrgica. Las sesiones en el laboratorio pueden utilizar objetos inanimados, o partes de animales.

Generalmente, los objetivos de aprendizaje en el laboratorio deben ser bien específicos. En estas sesiones el cirujano debe desarrollar la orientación espacial básica, y la habilidad de la coordinación ojo -mano, para esto puede utilizar material inanimado.

Otros objetivos del entrenamiento en el que se utiliza material inanimado son la práctica del aprendizaje de suturas intraabdominales en el entrenador pélvico y el desarrollar una familiaridad con las características del rayo láser.

Para aprender técnicas de resección y ablación de tejidos se utiliza órganos de animales como la vejiga del cerdo para entrenarse en ablación endometrial y otros procedimientos histeroscópicos, estos modelos tiene la desventaja que el sangrado intraoperatorio no ocurre y no se puede evaluar las habilidades hemostáticas.

La mayoría de los cursos de endoscopia operatoria combinan la enseñanza didáctica con el entrenamiento en el laboratorio.

Es importante que el estudiante no asuma, que con 1 o 2 días de práctica en laboratorio es suficiente para desarrollar una habilidad determinada.

Como se anota antes, en general las habilidades adquiridas en el laboratorio son bastante limitadas, y entrenamiento completo requiere de una observación continua de los procedimientos quirúrgicos actuales $\mathrm{y}$ de realizar los procedimientos bajo la supervisión de un tutor.

\section{Fase de observación}

Una vez adquirido el aprendizaje didáctico y de laboratorio, el estudiante procede a observar in vivo procedimientos de cirugía endoscópica, generalmente vía al monitor de video. La visión de procedimientos pregravados no es buena idea, porque el estudiante debe apreciar en forma completa la colocación y manipulación del instrumental endoscópico.

Idealmente, el estudiante debería observar entre 10 a 20 casos seleccionados por niveles de habilidades requeridas para su manejo, y además puede completar esta parte de su enseñanza gastando una o más semanas observando los procedimientos que realiza un cirujano endoscopista bien activo, o viendo casos unas dos veces por semana durante unos dos meses.

\section{Fase de práctica supervisada}

Después de haber observado 10 a 20 casos in vivo, de procedimientos específicos, el médico en entrenamiento está listo para iniciar sus propias cirugías endoscópicas, bajo la supervisión directa. El estudiante debe tener como meta el realizar por lo menos de 5 a 10 casos dentro de los niveles de habilidades deseados. El endoscopista supervisor debe tener gran experiencia en el campo y debe permitir al estudiante realizar toda o casi toda la cirugía según el grado de dificultad. 
El estudiante debe ser responsable por la selección pre quirúrgico del paciente, preparación, y manejo post-operatorio. Desafortunadamente, esta parte del proceso de entrenamiento es con frecuencia dejada de lado.

La enseñanza de la cirugía endoscópica debe ser idealmente parte del programa de entrenamiento durante la residencia de ginecología y obstetricia $(7,13-15)$.

El entrenamiento de residentes es el método más efectivo para resolver las numerosas discrepancias en los niveles de habilidades que se presentan con frecuencia entre los ginecólogos practicantes y los cirujanos generales. Infortunadamente, muchos programas de residencia no tienen en su currículum el desarrollo de este expertismo, dando como resultado la graduación de muchos obstetras ginecólogos o residentes de cirugía general con poco o ningún entrenamiento en cirugía endoscópica.

Los programas de entrenamiento deben intentar dar solución a esta deficiencia entrenando o reentrenado el cuerpo de profesores involucrados con la enseñanza de las diferentes técnicas quirúrgicas actuales.

Una aproximación similar a la sugerida previamente se debe tener en cuenta para acreditar el personal de los hospitales privados.

Como es pertinente en cualquier campo, las habilidades nuevas o adicionales deben ser a menudo adquiridas después del entrenamiento de la residencia. En este caso, el ginecólogo practicante debe proyectar su propio programa de entrenamiento.

El proceso completo de entrenamiento y certificación puede tomar de 2 a 3 meses para cada nivel de habilidades que va ser aprendido. Desafortunadamente, la demanda popular para este tipo de cirugía a aumentado exponencialmente, estimulando a muchos cirujanos a realizar procedimientos para los cuales no están familiarizados o entrenados, a menudo con pobres resultados, aumentando los costos quirúrgicos, elevando la morbimortalidad de los pacientes.

\section{Certificación y acreditación}

El certificado de capacidad (documentación del conocimiento y expertismo) no se puede extender, hasta que la persona en entrenamiento demuestre realmente al cirujano supervisor unas habilidades satisfactorias correspondientes al nivel de habilidades deseado.

$\mathrm{Si}$ el entrenamiento endoscópico es adquirido durante la residencia, la certificación del expertismo y habilidades son responsabilidad del director del programa, y la asignación de privilegios quirúrgicos hospitalarios es por tanto de rutina.
Alternativamente, si la mayor parte del entrenamiento es adquirido después de la residencia, el proceso de certificación y acreditación no esta bien definido.

Mientras un instructor de curso estará feliz de documentar a sus estudiantes o los tutores de calificar los casos supervisados por ellos, es improbable que uno u otro asuma la responsabilidad legal y ética de certificar la capacidad del individuo. Por tanto, la certificación del cirujano que adquirió su entrenamiento post residencia debe estar muy amarrada a la asignación de privilegios (acreditación) por el hospital donde labora.

El proceso de acreditación post residencia se puede basar en: (a) entrenamiento durante la residencia, (b) revisión de una lista de casos realizados durante 12 a 18 meses de practica, (c) documentación del entrenamiento realizado, incluyendo el observacional y el supervisado (tutorial).

Para minimizar la posibilidad de litigios legales, y en vista de que los procedimientos quirúrgicos endoscópicos son realizados con más frecuencia, este autor recomienda que los hospitales, EPS, IPS, implementen su propio sistema de acreditación ahora mismo.

Un método que La Sociedad Colombiana de Médicos Endoscopistas ha encontrado útil para iniciar el proceso de acreditación con mínimas repercusiones, ha sido utilizar los esquemas descritos en las tablas 1 y 2 por niveles de habilidades específicos. A los cirujanos ginecólogos endoscopistas que desean acreditación se les solicita, casos que puedan ilustrar su grado de experiencia y nivel de habilidades. Los casos deben cubrir un periodo de 12 a 18 meses de practica en cirugía ginecológica endoscópica, la información de los casos debe incluir nombre de la paciente, dirección, teléfono, indicación del procedimiento, patología, complicaciones, días de hospitalización.

Los casos son revisados por el comité permanente de acreditación, y él determina si el cirujano tiene suficiente experiencia (ej. número de casos) para el nivel de habilidades solicitado $\mathrm{y}$ si el número de complicaciones es significativo. Salvo cualquier problema obvio, como el no tener el número adecuado de casos, se otorgan los privilegios solicitados.

En el momento actual es indispensable tener e implementar un protocolo racional y bien estructurado de entrenamiento, certificación y acreditación en endoscopia quirúrgica ginecológica. $\mathrm{Si}$ nosotros no nos preocupamos como ginecólogos endoscopistas quirúrgicos practicantes de establecer las pautas, otras partes interesadas llámense gobierno, medicina prepagada, empresas prestadoras de servicios de salud, se encargaran de establecer los procedimientos unilateralmente. 


\section{BIBLIOGRAFIA}

1. Hulka JF, Peterson HB, Phillips JM, Surrey MW. Operative histeroscopy. American Association of Gynecologic Laparoscopists 1991, membership survey. J Reprod Med 1993; 3: 572-577.

2. Lehmann -Willenbrock E, Riedel HH, Mecke H, Semm K. Pelviscopy / Laparoscopy and its complications in Gemany, 1949 1988. J Reprod Med 1992; 7: 671-676.

3. Peterson HB, Hulka JF, Phillips JM, Surrey MW. Laparoscopic sterilization. American Association of Gynecologic Laparoscopists. 1991 membership. J Reprod Med 1993; 6: 574-579.

4. Riedel HH, Lehmann - Willenbrock E, Semm K. The development of pelviscopic (Iaparoscopic) surgical procedures in former East Germany - statistics from 1986 -1988 inclusive. Zentralbl-Gynakol 1993; 115: 210-220.

5. Azzis R. Operative endoscopy: the pressing need for a structured training an credentialing process. Fertil Steril. 1992; 58: 1100-1102.

6. Altman LK. Surgical injuries lead to new rule. New York Times. 1992; June 14, p 1.

7. Azzis R. Training, certification and credentialing in gynecologic operative endoscopy. Clin Obstet Gynecol. 1995; 38: 313-318.

8. The American College of Obstetrician and Gynecologists. Credentialing guidelines for new operative procedures. ACOG Committee Opinion 142, August 1994.
9. American Association of Gynecologic Laparoscopists. Credentialing guidelines for operative endoscopy. September 1992.

10. The American Collegue Of Obstetrician and Gynecologists. Credentialing guidelines for operative laparoscopy. ACOG Committee Opinion 106, April 1992.

11. The American Collegue of Obstetricians and Gynecologists. Credentialing guidelines for operative hysteroscopy. ACOG Committee Opinion 107, April 1992.

12. Society for Reproductive Surgeons - American Fertility Society. Guidelines for attaning privileges in gynecologic operative endoscopy. Fertil Steril. 1994; 621:118-119.

13. CREOG Trainining Guidelines: Advanced Surgical Techniques for Residency Training Programs in Obstetrics and Gynecology, 1994. A vailable through the Council on Resident Education in Obstetrics and Gynecology, 409 12th Street SW, Washington, DC 20024-2188.

14. Sammarco MJ, Youngblood JP. A resident teaching program in operative endoscopy. Obstet Gynecol. 1993; 81: 463-466.

15. Dent TL. Training, credaentialing, and evaluation in laparoscopy surgery. Surg Clin North Am 1992; 72: 1003-1011. 